\title{
Von Willebrand Disease in Pregnancy
}

\author{
${ }^{1}$ Rashmi Bagga, ${ }^{2}$ Akanksha Jangid, ${ }^{3}$ Shalini Gainder, ${ }^{4}$ Pankaj Malhotra, ${ }^{5}$ Subhash Varma \\ ${ }^{6}$ Jasmina Ahluwalia, ${ }^{7}$ Aashima Arora
}

\begin{abstract}
Von Willebrand disease (VWD) is the most common inherited bleeding disorder. It is due to deficiency of von Willebrand factor (vWF) which may be either quantitative or qualitative. There are 3 types of VWD out of which Type III is the rarest but the most severe. We report a 27 years second gravida diagnosed with vWD in her index pregnancy. Though her antenatal period was uneventful, she developed complications in the perioperative period following a caesarean section. However, with a multidisciplinary team effort, the maternal and fetal outcome were favourable.
\end{abstract}

Keywords: Von Willebrand disease, Pregnancy, Cesarean section.

How to cite this article: Bagga R, Jangid A, Gainder S, Malhotra P, Varma S, Ahluwalia J, Arora A. Von Willebrand Disease in Pregnancy. J Postgrad Med Edu Res 2014;48(3):157-158.

\section{Source of support: Nil}

\section{Conflict of interest: None}

\section{INTRODUCTION}

Von Willebrand disease (vWD) is the most common inherited bleeding disorder. It is due to deficiency of von Willebrand Factor (vWF) which may be either quantitative or qualitative. ${ }^{1}$ There are 3 types of vWD, all of which have autosomal inheritance. Types I and II have autosomal dominant inheritance while type III is an autosomal recessive disorder. Type III is the rarest and most severe of the three. Its prevalence in normal population is about $1 \%$ as per laboratory data but only one tenth of them have clinically significant bleeding manifestations. Women are more likely to be symptomatic as compared to men because of the haemostatic challenges of menstruation, pregnancy

\footnotetext{
${ }^{1,5}$ Professor, ${ }^{2,7}$ Senior Resident, ${ }^{3}$ Assistant Professor

${ }^{4,6}$ Additional Professor

1-3,7 Department of Obstetrics and Gynecology, Postgraduate Institute of Medical Education and Research, Chandigarh, India

${ }^{4,5}$ Internal Medicine, Postgraduate Institute of Medical Education and Research, Chandigarh, India

${ }^{6}$ Hematology, Postgraduate Institute of Medical Education and Research, Chandigarh, India

Corresponding Author: Rashmi Bagga, Professor, Department of Obstetrics and Gynecology, Postgraduate Institute of Medical Education and Research, Chandigarh, India, Phone: 0987249602, 09914209349, Fax: 0172-2744401, e-mail: rashmibagga@gmail.com
}

and parturition. ${ }^{2}$ In resource limited settings, Type III cases predominate as they are more likely to seek medical care. We report a case of a woman with Type III vWD diagnosed during her index pregnancy. Her antenatal and intrapartum management and the problems she faced during and after childbirth are discussed.

\section{CASE REPORT}

A 27-year-old female, second gravid with previous one cesarean delivery, presented at 18 weeks of gestation with bleeding from gums for 3 days. She had history of menorrhagia, prolonged bleeding from wound sites, excessive bleeding during her last cesarean delivery and had primary as well as secondary postpartum hemorrhage (PPH). However, none of the other family members over two generations had any bleeding diathesis.

Her laboratory evaluation showed a prolonged bleeding time: 23 minutes (normal: 2 to 8 minutes), and partial thromboplastin time with Kaolin (PTTK): 56 seconds (control: 25 to 40 seconds), with a normal prothrombin time: 14 seconds (control: $10-15$ seconds), platelet count: $230 \times 10^{9} / \mathrm{L}$ (normal: $\left.150-400 \times 10^{9} / 1\right)$. Ristocetin-induced platelet aggregation was markedly impaired with ristocetin $1.5 \mathrm{mg} / \mathrm{ml}$.

Factor VIII assay: 2\% (normal: 60-160\%), VWF antigen assay $\leq 1 \%$ (normal: $50-160 \%$ ) and ristocetin cofactor activity $<1 \%$ (normal: $50-200 \%$ ). A diagnosis of VWD type III was made.

Her antenatal period remained asymptomatic. She was admitted at $37+6$ weeks gestation for elective cesarean in view of previous cesarean and cephalopelvic disproportion. Calculated dose of VWF replacement was given 1 hour before surgery (patient's weight $60 \mathrm{~kg}$, factor requirement, $50 \mathrm{IU} \times 60=3000 \mathrm{IU}$, cost $-₹ 81,000$ ). Preoperatively, bleeding time was 3 minutes and PTTK was 29 seconds. Elective cesarean with bilateral tubal ligation was done. She delivered a baby boy weighing $2.234 \mathrm{~kg}$ with Apgar score of 8 and 9. Intraoperatively, she has excessive hemorrhage for which she received oxytocics and injection tranexamic acid. In postoperative period, she had continuous trickling of vaginal blood which was more than average but never massive. Bleeding was managed conservatively, and she received three blood transfusions. Due to nonaffordability for further factor replacement therapy, she received 5 units 
of cryoprecipitate daily for 3 days. Her bleeding time and PTTK normalized. No further complications were noticed and patient was discharged on postoperative day 11 . Neonate had no bleeding complication and remained asymptomatic till the day of discharge.

Patient again presented on day 56 postoperative with secondary PPH. This time, she received 19 cryoprecipitates, seven blood transfusions, tranexamic acid, and was started on oral progesterones (norethisterone $10 \mathrm{mg}$ tds). Bleeding stopped with conservative management, and patient was discharged under satisfactory conditions on tapering dose of norethisterone.

\section{DISCUSSION}

Von Willebrand disease presents unique challenges during pregnancy and childbirth. Ideally, women with VWD contemplating pregnancy should be counseled for the increased risk for bleeding complications during pregnancy and a significant risk of PPH. Before conception, she should receive genetic counseling regarding inheritance of the disease to the offspring. During pregnancy, levels of VWF and factor VIII increase, reaching peak at 34 weeks gestation and decrease in the postpartum period. Women with type I disease may achieve levels more than $50 \mathrm{IU} / \mathrm{dl}$, the lower limit of normal range (50-249\%), and usually manage to complete pregnancy without much complications. However, women with type III VWD experience no significant increase in VWF or factor VIII levels. In these patients, VWF/factor VIII level testing is recommended in every trimester and levels should be maintained above $50 \mathrm{IU} / \mathrm{dl}$ prior to delivery.

Regarding obstetric complications, $33 \%$ of these women experience first trimester bleeding and rate of miscarriage has been reported between 12 and $25 \%$, which is similar when compared to general population. Similarly, risk of antepartum hemorrhage (APH) is also not increased in them. These women are primarily at increased risk for primary and secondary PPH (20-29\%). ${ }^{3,4}$ The average time of presentation of postpartum hemorrhage is $15.7 \pm 5.2$ days after delivery. Perineal hematomas are also seen at increased frequency in these women ${ }^{5}(6 \%$ vs $0.22 \%)$. This implies that women with VWD should be frequently evaluated clinically and weekly postpartum visits are recommended. Infants with VWD are at increased risk for intracranial hemorrhage and scalp hematoma during labor and delivery, especially with the use of invasive fetal monitoring and instrumental delivery.

Management options of VWD in pregnancy include desmopressin, transfusional therapies and antifibrinolytic therapy. 1-desmopressin-8-arginine vasopressin (desmopressin acetate) is a synthetic vasopressin agonist. It belongs to category B. It promotes release of preformed VWF from endothelial cells and is most effective in patients with type I disease. However, in type III disease, as was in our patient, desmopressin acetate is ineffective.

The safety of these drugs during pregnancy and lactation is not established, though tranexamic acid has been used in placental abruption, cesarean and PPH without apparent maternal and fetal adverse effects.

Virally, inactivated VWF/factor VIII concentrates (plasma derived factor VIII/intermediate purity factor VIII) remain the treatment of choice for patients with VWD type III and those who are unresponsive to desmopressin acetate. Immunopurified or recombinant factor VIII contains little or no VWF and, thus, are of no use in VWD. The high cost of factor concentrates remains the limiting factor.

Cryoprecipitates, derived from plasma, is used under rare circumstances to treat VWD. However, cryoprecipitates do not undergo virucidal treatment and carry risk of transmission of viral and other blood-borne infections. The use of cryoprecipitate is strongly discouraged by the National Hemophilia Foundation, except in life or limb-threatening situations when no VWF concentrate is available. In developing countries, where either virally inactivated plasma concentrates are either not available or too expensive to afford, this may be the only option available.

\section{REFERENCES}

1. Kasper DL, Braunwald E, Fauci AS, Hauser SL, Longo DL, Lameson JL. Harrison's Principles of Internal Medicine. 16th ed. Disorders of Hemostasis 2004; p. 676-678.

2. Kujovich JL. Von Willebrand disease and pregnancy. J Thromb Haemost 2005;3(2):246-253.

3. James AH. Von Willebrand disease. Obstet Gynecol Surv 2006; 61(2):136-145.

4. Foster PA. The reproductive health of women with von Willebrand disease unresponsive to DDAVP: results of an international survey. Thromb Haemost 1995;74(2):784-790.

5. Greer IA, Lowe GD, Walker JJ, et al. Haemorrhagic problems in obstetrics and gynaecology in patients with congenital coagulopathies. Br J Obstet Gynaecol 1991;98(9):909-918. 